\title{
MULTILINEAR POLYNOMIALS ARE SURJECTIVE ON ALGEBRAS WITH SURJECTIVE INNER DERIVATIONS
}

\author{
DANIEL VITAS
}

\begin{abstract}
Let $f\left(X_{1}, \ldots, X_{n}\right)$ be a nonzero multilinear noncommutative polynomial. If $A$ is a unital algebra with a surjective inner derivation, then every element in $A$ can be written as $f\left(a_{1}, \ldots, a_{n}\right)$ for some $a_{i} \in A$.
\end{abstract}

\section{INTRODUCTION}

Let $F$ be a field. By $F\left\langle X_{1}, \ldots, X_{n}\right\rangle$ we denote the free algebra in $X_{i}$ over $F$; its elements are called noncommutative polynomials. Let $A$ be any algebra over $F$ and let $f=f\left(X_{1}, \ldots, X_{n}\right)$ be any noncommutative polynomial. The set

$$
f(A)=\left\{f\left(a_{1}, \ldots, a_{n}\right) \mid a_{1}, \ldots, a_{n} \in A\right\}
$$

is called the image of $f$. If $f(A)=A$, then $f$ is said to be surjective on $A$.

We say that $f$ is multilinear if it is of the form

$$
f\left(X_{1}, \ldots, X_{n}\right)=\sum_{\sigma \in S_{n}} \lambda_{\sigma} X_{\sigma(1)} \ldots X_{\sigma(n)}
$$

for some $\lambda_{\sigma} \in F$. The L'vov-Kaplansky conjecture states that if $F$ is an infinite field and $A=M_{n}(F)$ is the algebra of $n \times n$ matrices over $F$, then $f(A)$ is a vector space (as a matter of fact, it can only be one of the following four vector spaces: $\{0\}$, the space of scalar matrices $F 1$, the space of traceless matrices $[A, A]$, and $A$ ). In [5], Kanel-Belov, Malev, and Rowen confirmed this conjecture for the case where $n=2$ and $F$ is quadratically closed. Since then, there has been a lot of effort by several authors to solve the conjecture for larger $n$; however, even the $n=3$ case is currently only partially solved. Various variations of the problem have also been proposed and studied. We refer the reader to the recent survey article [6] for a thorough account of this line of investigation.

The initial idea, from which this paper arose, was to consider the infinite-dimensional version of the L'vov-Kaplansky conjecture. More specifically, if $V$ is an infinitedimensional vector space over $F$ and $A=\operatorname{End}_{F}(V)$, then one can ask whether $f(A)$ is a vector space for every multilinear polynomial $f$. We will see that this is indeed true. In fact, if $f \neq 0$ then $f(A)$ is simply equal to $A$, i.e., $f$ is surjective on $A$. We remark that the assumption that $f$ is multilinear is indeed necessary-see 2 , Example 3.16].

We will actually prove that this result, i.e., $f(A)=A$ with $f$ multilinear and nonzero, holds for a considerably larger class of algebras, which we now introduce. Recall that a map of the form $x \mapsto[v, x]$, where $v$ is a fixed element in $A$, is called an inner derivation of $A$ (here, $[v, x]$ stands for $v x-x v$ ). We will be interested in

\footnotetext{
Key words and phrases. Multilinear noncommutative polynomial, surjective inner derivation, L'vov-Kaplansky conjecture.

Mathematics Subject Classification (2020). 16R99, 16W25.
} 
algebras $A$ that have a surjective inner derivation. That is, there exists a $v \in A$ such that, for every $y \in A$, there is an $x \in A$ satisfying $[v, x]=y$.

As inner derivations have nontrivial kernels, no finite-dimensional algebra has a surjective inner derivation. Moreover, the same is true for any PI-algebra $A$, since $[A, A]$ is always a proper subset of $A$ [4]. Nevertheless, the class of algebras with surjective inner derivations is fairly large, as we will now show.

Examples 1.1. 1. Let $V$ be an infinite-dimensional vector space over a division algebra $D$ (over any field $F$ ), and let $A=\operatorname{End}_{D}(V)$. Let $\left\{e_{i, n} \mid(i, n) \in I \times \mathbb{N}\right\}$ be a basis of $V$ (here we used the standard fact that every infinite set $I$ has the same cardinality as $I \times \mathbb{N}$ ) and let $v \in A$ be given by $v\left(e_{i, n}\right)=e_{i, n+1}$. Take $y \in A$. Then $x \in A$ defined by $x\left(e_{i, 1}\right)=0$ and

$$
x\left(e_{i, n}\right)=-v^{n-2} y\left(e_{i, 1}\right)-v^{n-3} y\left(e_{i, 2}\right)-\cdots-v y\left(e_{i, n-2}\right)-y\left(e_{i, n-1}\right), \quad n \geq 2,
$$

satisfies $[v, x]=y$. Thus, $A$ is an algebra with a surjective inner derivation.

2. Let $\operatorname{char}(F)=0$. Suppose an algebra $A$ is generated by a pair of elements $v, w$ satisfying $[v, w]=1$ together with the elements that commute with both $v$ and $w$. It is easy to see that every element in $A$ is then a linear combination of elements of the form $v^{k} w^{\ell} t$ with $k, \ell \geq 0$ and $[t, v]=[t, w]=0$. Since $\left[v, \frac{1}{\ell+1} v^{k} w^{\ell+1} t\right]=v^{k} w^{\ell} t$, it follows that $[v, A]=A$, so $A$ is an algebra with a surjective inner derivation. Important concrete examples of such algebras $A$ are Weyl algebras $A_{n}(F), n \in \mathbb{N}$.

3. From the paper by Cohn [3] it is evident that there exist division algebras with surjective inner derivations.

4. The direct product of any family, finite or infinite, of algebras with surjective inner derivations is an algebra with a surjective inner derivation.

5. A homomorphic image of an algebra with a surjective inner derivation is again an algebra with a surjective inner derivation.

6. If $A$ is an algebra with a surjective inner derivation and $B$ is any unital algebra, then $A \otimes B$ is an algebra with a surjective inner derivation. Indeed, if $x \mapsto[v, x]$ is a surjective inner derivation of $A$, then $x \mapsto[v \otimes 1, x]$ is a surjective inner derivation of $A \otimes B$.

We will establish the following theorem.

Theorem 1.2. If $A$ is a unital algebra with a surjective inner derivation, then every nonzero multilinear polynomial is surjective on $A$.

Let us point out the two most prominent examples to which this theorem applies.

Corollary 1.3. Let $V$ be an infinite-dimensional vector space over a division algebra $D$ (over any field $F$ ). Then every nonzero multilinear polynomial is surjective on the algebra $\operatorname{End}_{D}(V)$.

Corollary 1.4. Every nonzero multilinear polynomial is surjective on the nth Weyl algebra $A_{n}(F)$ (here, $F$ is any field with characteristic 0).

We will actually prove a more general result, Theorem 4.7 which involves somewhat more general polynomials (which we call admissible partially commutative polynomials) and requires less than the surjectivity of a derivation. What follows is devoted to its proof. More precisely, in Section 2 we provide an appropriate setting for our problem, in Section 3 we consider a special system of linear equations that occurs in the proof, and in Section 4 we give the proof of the main result. 


\section{Admissible partially commutative polynomials}

Let $F$ be a field. By

$$
F\left\langle X_{1}, \ldots, X_{n}\right\rangle \amalg F[U, V]
$$

we denote the coproduct (see, e.g., [1, Section 1.4]) of the free algebra $F\left\langle X_{1}, \ldots, X_{n}\right\rangle$, i.e., the algebra of polynomials in noncommuting variables $X_{i}$, and $F[U, V]$, the algebra of polynomials in two commuting variables $U$ and $V$. We will refer to the elements of this coproduct algebra as partially commutative polynomials. We may think of them as polynomials in $X_{i}, U, V$ where the variables $U$ and $V$ commute among themselves, but do not commute with the variables $X_{i}$.

Let $A$ be a unital algebra over $F$ and let $v$ be a fixed element in $A$. In Section 4 we will impose some conditions on $v$, but at this stage it can be any element. By $F[v]$ we denote the (unital) subalgebra of $A$ generated by $v$. Take any $x_{1}, \ldots, x_{n} \in A$ and $u \in F[v]$, and let

$$
\operatorname{Ev}_{x_{1}, \ldots, x_{n} ; u}: F\left\langle X_{1}, \ldots, X_{n}\right\rangle \amalg F[U, V] \rightarrow A
$$

be the algebra homomorphism sending $X_{i}$ to $x_{i}, U$ to $u$, and $V$ to $v$. Note that $\mathrm{Ev}_{x_{1}, \ldots, x_{n} ; u}$ extends the usual evaluation homomorphisms on $F\left\langle X_{1}, \ldots, X_{n}\right\rangle$ and $F[U, V]$, respectively, so its existence follows from the standard properties of the coproduct. We define the image of a partially commutative polynomial

$$
f \in F\left\langle X_{1}, \ldots, X_{n}\right\rangle \amalg F[U, V]
$$

as

$$
f(A ; v)=\left\{\operatorname{Ev}_{x_{1}, \ldots, x_{n} ; u}(f) \mid x_{1}, \ldots, x_{n} \in A, u \in F[v]\right\} .
$$

If $f$ does not involve the variable $U$, we will write

$$
\operatorname{Ev}_{x_{1}, \ldots, x_{n} ;}(f)
$$

instead of $\operatorname{Ev}_{x_{1}, \ldots, x_{n} ; u}(f)$. Of course, if $f$ is a noncommutative polynomial, i.e, an element of the subalgebra $F\left\langle X_{1}, \ldots, X_{n}\right\rangle$ of $F\left\langle X_{1}, \ldots, X_{n}\right\rangle \amalg F[U, V]$, then $f(A)=$ $f(A ; v)$. (We actually will not deal with images of partially commutative polynomials until Section 4, but giving this definition at this early stage may help the reader to understand the context.)

The reason for introducing partially commutative polynomials is the method of our proof. In order to get closer to "pure" noncommutative polynomials (i.e., elements of $\left.F\left\langle X_{1}, \ldots, X_{n}\right\rangle\right)$ in which we are primarily interested, we will, in the course of proof, often substitute $V^{k}$ for $U$. Let

$$
\pi_{k}: F\left\langle X_{1}, \ldots, X_{n}\right\rangle \amalg F[U, V] \rightarrow F\left\langle X_{1}, \ldots, X_{n}\right\rangle \amalg F[U, V]
$$

be the homomorphism that fixes each $X_{i}$ and $V$, and sends $U$ to $V^{k}$. A routine proof shows that

$$
\pi_{k}(f)(A ; v) \subseteq f(A ; v)
$$

for every $f \in F\left\langle X_{1}, \ldots, X_{n}\right\rangle \amalg F[U, V]$ and every $k \in \mathbb{N}_{0}=\mathbb{N} \cup\{0\}$.

We need some more notation. First of all, we will write

$$
\left[x_{1}, x_{2}, \ldots, x_{n}\right]=\left[x_{1},\left[x_{2}, \ldots, x_{n}\right]\right]
$$

and

$$
\left[x_{1}, x_{2}\right]_{k}=[\underbrace{x_{1}, \ldots, x_{1}}_{k}, x_{2}] .
$$


For any $r \in \mathbb{N}_{0}$, we define

$$
\mathscr{B}_{r}=\left\{\mathbf{b}=\left(\mathbf{b}_{1}, \ldots, \mathbf{b}_{n}\right) \in \mathbb{N}_{0}^{n} \mid \sum_{i=1}^{n} \mathbf{b}_{i}=r\right\},
$$

and for any $\mathbf{b} \in \mathscr{B}_{r}$ we define

$$
X_{j}^{\mathbf{b}}=[\underbrace{V, \ldots, V}_{\mathbf{b}_{j}}, X_{j}]=\left[V, X_{j}\right]_{\mathbf{b}_{j}} ;
$$

if $\mathbf{b}_{j}=0$, it should be understood that $X_{j}^{\mathbf{b}}=X_{j}$. For $i \in\{1, \ldots, n\}$, we define

$$
X_{j}^{\mathbf{b}, i}=\left\{\begin{array}{l}
{\left[U, X_{j}^{\mathbf{b}}\right], j=i} \\
X_{j}^{\mathbf{b}}, j \neq i
\end{array} .\right.
$$

We extend both definitions by setting

$$
\begin{aligned}
\left(X_{j_{1}} X_{j_{2}} \ldots X_{j_{k}}\right)^{\mathbf{b}} & =X_{j_{1}}^{\mathbf{b}} X_{j_{2}}^{\mathbf{b}} \ldots X_{j_{k}}^{\mathbf{b}}, \\
\left(X_{j_{1}} X_{j_{2}} \ldots X_{j_{k}}\right)^{\mathbf{b}, i} & =X_{j_{1}}^{\mathbf{b}, i} X_{j_{2}}^{\mathbf{b}, i} \ldots X_{j_{k}}^{\mathbf{b}, i},
\end{aligned}
$$

for all $j_{1}, \ldots, j_{k} \in\{1, \ldots, n\}$.

We are now in a position to define the polynomials that will play a central role in this paper.

Definition 2.1. A partially commutative polynomial

$$
f \in F\left\langle X_{1}, \ldots, X_{n}\right\rangle \amalg F[U, V]
$$

is said to be admissible if there exists an $r \in \mathbb{N}_{0}$ (which we call the order of $f$ ) such that $f$ is either of the form

$$
f=\sum_{\sigma \in S_{n}} \sum_{\mathbf{b} \in \mathscr{B}_{r}} \lambda_{\sigma}^{\mathbf{b}}\left(X_{\sigma(1)} X_{\sigma(2)} \ldots X_{\sigma(n)}\right)^{\mathbf{b}}
$$

for some $\lambda_{\sigma}^{\mathbf{b}} \in F$ or of the form

$$
f=\sum_{\sigma \in S_{n}} \sum_{\mathbf{b} \in \mathscr{B}_{r}} \sum_{i=1}^{n} \lambda_{\sigma, i}^{\mathbf{b}}\left(X_{\sigma(1)} X_{\sigma(2)} \ldots X_{\sigma(n)}\right)^{\mathbf{b}, \sigma(i)}
$$

for some $\lambda_{\sigma, i}^{\mathbf{b}} \in F$. If (2.2) holds, then $f$ is said to be of type one, and if (2.3) holds, then $f$ is said to be of type two.

Remark 2.2. In the case where $r=0, \mathscr{B}_{r}$ consists only of the sequence of zeros $\mathbf{b}$. Therefore, in this case we have

$$
\left(X_{\sigma(1)} X_{\sigma(2)} \ldots X_{\sigma(n)}\right)^{\mathbf{b}}=X_{\sigma(1)} X_{\sigma(2)} \ldots X_{\sigma(n)} .
$$

Thus, admissible partially commutative polynomials of type one and of order 0 are of the form

$$
f=\sum_{\sigma \in S_{n}} \lambda_{\sigma} X_{\sigma(1)} X_{\sigma(2)} \ldots X_{\sigma(n)},
$$

so these are exactly the multilinear noncommutative polynomials. This is of crucial importance for us: any result on admissible partially commutative polynomials yields a result on multilinear noncommutative polynomials.

To gain some feeling for the notions we have defined, we record a simple example. 
Example 2.3. Let us describe admissible partially commutative polynomials in $F\left\langle X_{1}, X_{2}\right\rangle \amalg F[U, V]$ of order 1 . We have

$$
\mathscr{B}_{1}=\{(1,0),(0,1)\} .
$$

Note that

$$
\begin{array}{ll}
\left(X_{1} X_{2}\right)^{(1,0)}=\left[V, X_{1}\right] X_{2}, \quad & \left(X_{2} X_{1}\right)^{(1,0)}=X_{2}\left[V, X_{1}\right], \\
\left(X_{1} X_{2}\right)^{(0,1)}=X_{1}\left[V, X_{2}\right], & \left(X_{2} X_{1}\right)^{(0,1)}=\left[V, X_{2}\right] X_{1},
\end{array}
$$

and thus

$$
\begin{array}{ll}
\left(X_{1} X_{2}\right)^{(1,0), 1}=\left[U, V, X_{1}\right] X_{2}, & \left(X_{2} X_{1}\right)^{(1,0), 1}=X_{2}\left[U, V, X_{1}\right], \\
\left(X_{1} X_{2}\right)^{(0,1), 1}=\left[U, X_{1}\right]\left[V, X_{2}\right], & \left(X_{2} X_{1}\right)^{(0,1), 1}=\left[V, X_{2}\right]\left[U, X_{1}\right], \\
\left(X_{1} X_{2}\right)^{(1,0), 2}=\left[V, X_{1}\right]\left[U, X_{2}\right], & \left(X_{2} X_{1}\right)^{(1,0), 2}=\left[U, X_{2}\right]\left[V, X_{1}\right], \\
\left(X_{1} X_{2}\right)^{(0,1), 2}=X_{1}\left[U, V, X_{2}\right], & \left(X_{2} X_{1}\right)^{(0,1), 2}=\left[U, V, X_{2}\right] X_{1} .
\end{array}
$$

Hence, admissible polynomials of type two are of the form

$$
\begin{aligned}
f & =\lambda_{\mathrm{id}, 1}^{(1,0)}\left[U, V, X_{1}\right] X_{2}+\lambda_{(12), 2}^{(1,0)} X_{2}\left[U, V, X_{1}\right] \\
& +\lambda_{\mathrm{id}, 1}^{(0,1)}\left[U, X_{1}\right]\left[V, X_{2}\right]+\lambda_{(12), 2}^{(0,1)}\left[V, X_{2}\right]\left[U, X_{1}\right] \\
& +\lambda_{\mathrm{id}, 2}^{(1,0)}\left[V, X_{1}\right]\left[U, X_{2}\right]+\lambda_{(12), 1}^{(1,0)}\left[U, X_{2}\right]\left[V, X_{1}\right] \\
& +\lambda_{\mathrm{id}, 2}^{(0,1)} X_{1}\left[U, V, X_{2}\right]+\lambda_{(12), 1}^{(0,1)}\left[U, V, X_{2}\right] X_{1},
\end{aligned}
$$

while admissible polynomials of type one are of the form

$$
\begin{aligned}
f & =\lambda_{\mathrm{id}}^{(1,0)}\left[V, X_{1}\right] X_{2}+\lambda_{(12)}^{(1,0)} X_{2}\left[V, X_{1}\right] \\
& +\lambda_{\mathrm{id}}^{(0,1)} X_{1}\left[V, X_{2}\right]+\lambda_{(12)}^{(0,1)}\left[V, X_{2}\right] X_{1} .
\end{aligned}
$$

By definition, the vector space of admissible partially commutative polynomials of type two (resp. type one) is linearly spanned by the polynomials $\left(X_{\sigma(1)} \ldots X_{\sigma(n)}\right)$ b, $\sigma(i)$ (resp. $\left(X_{\sigma(1)} \ldots X_{\sigma(n)}\right)^{\mathbf{b}}$ ). Our goal now is to show that they are linearly independent and so they actually form a basis. To this end, we define

$$
B_{1}=\left\{X_{j_{1}} \ldots X_{j_{d}} \mid d \in \mathbb{N}, j_{1}, \ldots, j_{d} \in\{1, \ldots, n\}\right\}
$$

and

$$
B_{2}=\left\{U^{k} V^{l} \mid k, l \in \mathbb{N}_{0}, k+l>0\right\} .
$$

Of course, $B_{1} \cup\{1\}$ is the standard basis of $F\left\langle X_{1}, \ldots, X_{n}\right\rangle$ and $B_{2} \cup\{1\}$ is the standard basis of $F[U, V]$. Letting $M$ be the set of all alternating monomials from $B_{1}$ and $B_{2}$, we see from [1, Lemma 1.4.5] that $M \cup\{1\}$ is a basis of the vector space

$$
F\left\langle X_{1}, \ldots, X_{n}\right\rangle \amalg F[U, V]
$$

of partially commutative polynomials. Using this, it is easy to see that the eight (resp. four) polynomials occurring in Example 2.3 are linearly independent. We now proceed to the general case.

Proposition 2.4. For any $n \in \mathbb{N}$ and $r \in \mathbb{N}_{0}$,

$$
\left\{\left(X_{\sigma(1)} X_{\sigma(2)} \ldots X_{\sigma(n)}\right)^{\boldsymbol{b}} \mid \sigma \in S_{n}, \boldsymbol{b} \in \mathscr{B}_{r}\right\}
$$


and

$$
\left\{\left(X_{\sigma(1)} X_{\sigma(2)} \ldots X_{\sigma(n)}\right)^{\boldsymbol{b}, \sigma(i)} \mid \sigma \in S_{n}, \boldsymbol{b} \in \mathscr{B}_{r}, i=1, \ldots, n\right\}
$$

are linearly independent sets.

Proof. We will prove the linear independence of the second set; the proof for the first set is similar. Suppose

$$
\sum_{\sigma \in S_{n}} \sum_{\mathbf{b} \in \mathscr{B}_{r}} \sum_{i=1}^{n} \lambda_{\sigma, i}^{\mathbf{b}}\left(X_{\sigma(1)} X_{\sigma(2)} \ldots X_{\sigma(n)}\right)^{\mathbf{b}, \sigma(i)}=0
$$

where some of the scalars $\lambda_{\sigma, i}^{\mathbf{b}}$ are nonzero. Without loss of generality, we may assume that the set

$$
\mathcal{L}=\left\{(\mathbf{b}, i) \in \mathscr{B}_{r} \times\{1, \ldots, n\} \mid \lambda_{\mathrm{id}, i}^{\mathbf{b}} \neq 0\right\}
$$

is nonempty. Define the following strict total ordering on $\mathcal{L}$ :

$$
(\mathbf{b}, i) \prec\left(\mathbf{b}^{\prime}, i^{\prime}\right)
$$

$$
\Longleftrightarrow i<i^{\prime} \text { or }\left(i=i^{\prime} \text { and } \mathbf{b}_{j}<\mathbf{b}_{j}^{\prime} \text { for the least } j \text { satisfying } \mathbf{b}_{j} \neq \mathbf{b}_{j}^{\prime}\right) \text {. }
$$

Let $(\widetilde{\mathbf{b}}, \widetilde{i})$ be a maximal element of $\mathcal{L}$ with respect to $\prec$. We claim that by rewriting the above summation as a linear combination of elements of $M$, one arrives at a contradiction that $\lambda_{\mathrm{id}, \tilde{i}}^{\widetilde{\mathbf{b}}}=0$. The formal proof is a bit tedious but elementary, so we omit the details.

\section{A CERTAin System of LineAR EQUATiOns}

Let $\sigma \in S_{n}$ and let $r \in \mathbb{N}_{0}$. The purpose of this section is to examine the following system of linear equations:

$$
\sum_{i=1}^{n} \sum_{\mathbf{c} \in \mathscr{C}_{\sigma, k, i}} \alpha_{\sigma, i}^{\mathbf{c}} \mu_{i}^{\mathbf{b}-\mathbf{c}}=0
$$

for every $k \in \mathbb{N}$ and every $\mathbf{b} \in \mathscr{B}_{r+k}$, where

$$
\begin{gathered}
\mathscr{C}_{\sigma, k, i}=\left\{\mathbf{c}=\left(\mathbf{c}_{1}, \ldots, \mathbf{c}_{n}\right) \in \mathbb{N}_{0}^{n} \mid \mathbf{c}_{\sigma(1)}=\ldots=\mathbf{c}_{\sigma(i-1)}=0, \mathbf{c}_{\sigma(i)} \geq 1, \sum_{j=1}^{n} \mathbf{c}_{j}=k\right\}, \\
\alpha_{\sigma, i}^{\mathbf{c}}=\left(\begin{array}{c}
\sum_{j=1}^{n} \mathbf{c}_{j} \\
\mathbf{c}_{\sigma(i)}, \ldots, \mathbf{c}_{\sigma(n)}
\end{array}\right)=\frac{\left(\sum_{j=1}^{n} \mathbf{c}_{j}\right) !}{\mathbf{c}_{\sigma(i)} ! \ldots \mathbf{c}_{\sigma(n)} !} \in \mathbb{N},
\end{gathered}
$$

and $\mu_{i}^{\mathbf{b}^{\prime}}, \mathbf{b}^{\prime} \in \mathscr{B}_{r}, i=1, \ldots, n$, are the unknowns, with

$$
\mu_{i}^{\mathbf{b}^{\prime}}=0 \text { whenever } \mathbf{b}^{\prime} \in \mathbb{Z}^{n} \backslash \mathscr{B}_{r} .
$$

The only property of the scalars $\alpha_{\sigma, i}^{\mathbf{c}}$ that we actually need is that

$$
\alpha_{\sigma, i}^{k \mathbf{e}_{\sigma(i)}}=1,
$$

where

$$
\mathbf{e}_{j}=(0, \ldots, 0,1,0, \ldots, 0)
$$

with 1 appearing at the $j$ th place. 
The sole reason for discussing the system of equations (3.1) is that it will occur in the proof of the main theorem. We have introduced it in the exact form as needed. However, it is clear that there is no loss of generality in assuming that

$$
\sigma=\mathrm{id}
$$

We will write $\alpha_{i}^{\mathbf{c}}$ for $\alpha_{\text {id }, i}^{\mathbf{c}}$ and $\mathscr{C}_{k, i}$ for $\mathscr{C}_{\mathrm{id}, k, i}$.

The goal of this section is to show that our system of equations has only trivial solution $\mu_{i}^{\mathbf{b}^{\prime}}=0$ for all $\mathbf{b}^{\prime} \in \mathscr{B}_{r}, i=1, \ldots, n$. We illustrate our method of solving by a simple example.

Example 3.1. Let $n=2$ and $r=1$. We have $\mathscr{B}_{1}=\{(1,0),(0,1)\}$. Thus, our unknowns are $\mu_{1}^{(1,0)}, \mu_{1}^{(0,1)}, \mu_{2}^{(1,0)}$, and $\mu_{2}^{(0,1)}$.

First, let $k=1$. Note that $\mathscr{B}_{2}=\{(2,0),(1,1),(0,2)\}, \mathscr{C}_{1,1}=\{(1,0)\}, \mathscr{C}_{1,2}=$ $\{(0,1)\}$ and $\alpha_{1}^{(1,0)}=\alpha_{2}^{(0,1)}=1$. Thus, we have the equations

$$
\begin{aligned}
\mu_{1}^{(1,0)}+\mu_{2}^{(2,-1)} & =0, \\
\mu_{1}^{(0,1)}+\mu_{2}^{(1,0)} & =0, \\
\mu_{1}^{(-1,2)}+\mu_{2}^{(0,1)} & =0,
\end{aligned}
$$

for $b=(2,0),(1,1),(0,2)$, respectively, with $\mu_{2}^{(2,-1)}=\mu_{1}^{(-1,2)}=0$. By adding the equation $\mu_{1}^{(2,-1)}+\mu_{2}^{(3,-2)}=0$, which trivially holds since $\mu_{1}^{(2,-1)}=\mu_{2}^{(3,-2)}=0$, we extend the above system to

$$
\begin{gathered}
\mu_{1}^{(2,-1)}+\mu_{2}^{(3,-2)}=0, \\
\mu_{1}^{(1,0)}+\mu_{2}^{(2,-1)}=0, \\
\mu_{1}^{(0,1)}+\mu_{2}^{(1,0)}=0, \\
\mu_{1}^{(-1,2)}+\mu_{2}^{(0,1)}=0 .
\end{gathered}
$$

Now, let $k=2$. Note that $\mathscr{B}_{3}=\{(3,0),(2,1),(1,2),(0,3)\}, \mathscr{C}_{2,1}=\{(2,0),(1,1)\}$ and $\mathscr{C}_{2,2}=\{(0,2)\}$. Also, $\alpha_{1}^{(2,0)}=1, \alpha_{1}^{(1,1)}=2$ and $\alpha_{2}^{(0,2)}=1$. Thus, we have the equations

$$
\begin{gathered}
\mu_{1}^{(1,0)}+2 \mu_{1}^{(2,-1)}+\mu_{2}^{(3,-2)}=0, \\
\mu_{1}^{(0,1)}+2 \mu_{1}^{(1,0)}+\mu_{2}^{(2,-1)}=0, \\
\mu_{1}^{(-1,2)}+2 \mu_{1}^{(0,1)}+\mu_{2}^{(1,0)}=0, \\
\mu_{1}^{(-2,3)}+2 \mu_{1}^{(-1,2)}+\mu_{2}^{(0,1)}=0,
\end{gathered}
$$

for $b=(3,0),(2,1),(1,2),(0,3)$, respectively, with $\mu_{1}^{(-2,3)}=0$.

By substracting each line of the system (3.2) from the corresponding line in (3.3), we obtain

$$
\begin{aligned}
\mu_{1}^{(1,0)}+\mu_{1}^{(2,-1)} & =0, \\
\mu_{1}^{(0,1)}+\mu_{1}^{(1,0)} & =0, \\
\mu_{1}^{(-1,2)}+\mu_{1}^{(0,1)} & =0, \\
\mu_{1}^{(-2,3)}+\mu_{1}^{(-1,2)} & =0 .
\end{aligned}
$$


Since $\mu_{1}^{(2,-1)}=0$, the first equation yields $\mu_{1}^{(1,0)}=0$. Accordingly, the second equation gives $\mu_{1}^{(0,1)}=0$. From (3.2) we infer that $\mu_{2}^{(1,0)}=\mu_{2}^{(0,1)}=0$. We have thus shown that (3.1) has only trivial solution for $n=2$ and $r=1$.

As we can see from Example 3.1, the number of equations is different for different $k$. We remedy this by adding trivial equations. That is, we extend the system (3.1) to

$$
\sum_{i=1}^{n} \sum_{\mathbf{c} \in \mathscr{C}_{k, i}} \alpha_{i}^{\mathbf{c}} \mu_{i}^{\mathbf{b}-\mathbf{c}}=0
$$

for every $k \in \mathbb{N}$ and every $\mathbf{b} \in \mathbb{Z}^{n}$.

Let us check that any solution of the restricted system of equations (3.1) is also a solution of the extended system (3.5). For this purpose, we fix $k \in \mathbb{N}_{0}$ and $\mathbf{b} \in$ $\mathbb{Z}^{n} \backslash \mathscr{B}_{r+k}$, i.e., $\mathbf{b} \in \mathbb{Z}^{n}$ and either $\sum_{j=1}^{n} \mathbf{b}_{j} \neq r+k$ or $\mathbf{b}_{j}<0$ for some $j$. If $\sum_{j=1}^{n} \mathbf{b}_{j} \neq r+k$, then $\sum_{j=1}^{n}(\mathbf{b}-\mathbf{c})_{j} \neq r$ for all $\mathbf{c} \in \mathscr{C}_{k, i}$, since $\sum_{j=1}^{n} \mathbf{c}_{j}=k$. Thus, $\mathbf{b}-\mathbf{c} \notin \mathscr{B}_{r}$ for all $\mathbf{c} \in \mathscr{C}_{k, i}$; hence, all $\mu_{i}^{\mathbf{b}-\mathbf{c}}$ are zero and (3.5) holds. Similarly, we arrive at the same conclusion in the case where $\mathbf{b}_{j}<0$ for some $j$.

Let $V$ be the $F$-vector space with basis $\left\{M_{i}^{\mathbf{b}} \mid \mathbf{b} \in \mathbb{Z}^{n}, i=1, \ldots, n\right\}$ (here, $M_{i}^{\mathbf{b}}$ are just abstract symbols). For every $j=1, \ldots, n$, let $\ell_{j}: V \rightarrow V$ be the linear map given by

$$
\ell_{j}: M_{i}^{\mathbf{b}} \mapsto M_{i}^{\mathbf{b}-\mathbf{e}_{j}} .
$$

Note that the maps $\ell_{j}$ commute among themselves.

We make a connection between $M_{i}^{\mathbf{b}}$ and $\mu_{i}^{\mathbf{b}}$ through the linear functional $\phi: V \rightarrow$ $F$ given by

$$
\phi\left(M_{i}^{\mathbf{b}}\right)=\mu_{i}^{\mathbf{b}} .
$$

We can rewrite the system (3.5) as

$$
\sum_{i=1}^{n} \sum_{\mathbf{c} \in \mathscr{C}_{k, i}} \alpha_{i}^{\mathbf{c}} M_{i}^{\mathbf{b}-\mathbf{c}} \in \operatorname{ker} \phi
$$

for every $k \in \mathbb{N}$ and every $\mathbf{b} \in \mathbb{Z}^{n}$. Since $\mathbf{c} \in \mathscr{C}_{k, i}$ can be written as $\mathbf{c}=\mathbf{c}_{i} \mathbf{e}_{i}+\cdots+$ $\mathbf{c}_{n} \mathbf{e}_{n}$, we have $M_{i}^{\mathbf{b}-\mathbf{c}}=\ell_{i}^{\mathbf{c}_{i}} \ldots \ell_{n}^{\mathbf{c}_{n}} M_{i}^{\mathbf{b}}$. Thus, we can further rewrite our system as

$$
\sum_{i=1}^{n}\left(\sum_{\mathbf{c} \in \mathscr{C}_{k, i}} \alpha_{i}^{\mathbf{c}_{i}} \ell_{i}^{\mathbf{c}_{i}} \ldots \ell_{n}^{\mathbf{c}_{n}}\right) M_{i}^{\mathbf{b}} \in \operatorname{ker} \phi
$$

for every $k \in \mathbb{N}$ and every $\mathbf{b} \in \mathbb{Z}^{n}$. The expression in parentheses can be viewed as a (commutative) polynomial evaluated in $\ell_{i}, \ldots, \ell_{n}$. Since $\alpha_{i}^{k \mathbf{e}_{i}}=1$ and $\sum_{j=1}^{n} \mathbf{c}_{j}=k$ for every $\mathbf{c} \in \mathscr{C}_{k, i}$, the degree of this polynomial in the $i$ th variable is exactly $k$. This motivates the following definition.

Definition 3.2. Let $U$ be a subspace of $V$ and let $i_{1}, \ldots, i_{l} \in\{1, \ldots, n\}$ be distinct. We say that $M_{i_{1}}^{\mathbf{b}}, \ldots, M_{i_{l}}^{\mathbf{b}}$ satisfy a recurrence relation of order $k \in \mathbb{N}$ if

$$
\sum_{i \in\left\{i_{1}, \ldots, i_{l}\right\}} f_{i}\left(\ell_{i}, \ldots, \ell_{n}\right) M_{i}^{\mathbf{b}} \in U
$$

holds for every $\mathbf{b} \in \mathbb{Z}^{n}$ and some polynomials $f_{i} \in F\left[W_{i}, \ldots, W_{n}\right]$ such that the degree of $f_{i}$ in $W_{i}$ is $k$. 
We summarize our observations up to this point. Suppose $\mu_{i}^{\mathbf{b}} \in F$ satisfy (3.5), where $\mu_{i}^{\mathbf{b}}=0$ for all $\mathbf{b} \notin \mathscr{B}_{r}, i=1, \ldots, n$. By taking $U=\operatorname{ker} \phi$ with $\phi$ defined above, the elements $M_{1}^{\mathbf{b}}, \ldots, M_{n}^{\mathbf{b}}$ satisfy a recurrence relation of order $k$ for each $k \in \mathbb{N}$. Since only finitely many $\mu_{i}^{\mathbf{b}}$ can be nonzero, $M_{i}^{\mathbf{b}} \in U$ holds for all but finitely many elements. We claim that this already implies that $U=V$ or, equivalently, $\mu_{i}^{\mathbf{b}}=0$ for all $\mathbf{b} \in \mathscr{B}_{r}, i=1, \ldots, n$. To this end, we need a few lemmas.

Lemma 3.3. Let $U$ be a subspace of $V$. If $f_{i} \in F\left[W_{i}, \ldots, W_{n}\right]$ satisfy

$$
\sum_{i \in\left\{i_{1}, \ldots, i_{l}\right\}} f_{i}\left(\ell_{i}, \ldots, \ell_{n}\right) M_{i}^{b} \in U
$$

for every $\boldsymbol{b} \in \mathbb{Z}^{n}$, then

$$
\sum_{i \in\left\{i_{1}, \ldots, i_{l}\right\}} g\left(\ell_{1}, \ldots, \ell_{n}\right) f_{i}\left(\ell_{i}, \ldots, \ell_{n}\right) M_{i}^{b} \in U
$$

for every $\boldsymbol{b} \in \mathbb{Z}^{n}$ and every $g \in F\left[W_{1}, \ldots, W_{n}\right]$.

Proof. Since $U$ is a vector space, it suffices to consider the case where $g$ is a monomial, so

$$
g\left(\ell_{1}, \ldots, \ell_{n}\right)=\ell_{1}^{j_{1}} \ldots \ell_{n}^{j_{n}} .
$$

Take $\mathbf{b} \in \mathbb{Z}^{n}$. Using the commutativity of the $\ell_{j}$ s, we have

$$
\begin{aligned}
\sum_{i \in\left\{i_{1}, \ldots, i_{l}\right\}} g\left(\ell_{1}, \ldots, \ell_{n}\right) f_{i}\left(\ell_{i}, \ldots, \ell_{n}\right) M_{i}^{\mathbf{b}} & =\sum_{i \in\left\{i_{1}, \ldots, i_{l}\right\}} f_{i}\left(\ell_{i}, \ldots, \ell_{n}\right) \ell_{1}^{j_{1}} \ldots \ell_{n}^{j_{n}} M_{i}^{\mathbf{b}} \\
& =\sum_{i \in\left\{i_{1}, \ldots, i_{l}\right\}} f_{i}\left(\ell_{i}, \ldots, \ell_{n}\right) M_{i}^{\mathbf{b}-j_{1} \mathbf{e}_{1}-\cdots-j_{n} \mathbf{e}_{n}} .
\end{aligned}
$$

Since (3.6) holds for $\mathbf{b}^{\prime}=\mathbf{b}-j_{1} \mathbf{e}_{1}-\cdots-j_{n} \mathbf{e}_{n}$, the desired conclusion follows.

The next lemma generalizes the process of subtracting equations in Example 3.1 (and is essentially a version of Gaussian elimination).

Lemma 3.4. Let $U$ be a subspace of $V$ and let $1 \leq i_{1}<\cdots<i_{l} \leq n$. Suppose $M_{i_{1}}^{b}, \ldots, M_{i_{l}}^{b}$ satisfy $l$ recurrence relations of order $k_{1}>\cdots>k_{l}$. Then $M_{i_{1}}^{b}$ satisfy a recurrence relation (of order $k_{1}$ ).

Proof. We proceed by induction on $l$. There is nothing to prove if $l=1$, so assume that $l>1$ and that the lemma is true for all numbers smaller than $l$. By assumption,

$$
\sum_{i \in\left\{i_{1}, \ldots, i_{l}\right\}} f_{i j}\left(\ell_{i}, \ldots, \ell_{n}\right) M_{i}^{\mathbf{b}} \in U
$$

for every $\mathbf{b} \in \mathbb{Z}^{n}$ and every $j=1, \ldots, l$, where $f_{i j} \in F\left[W_{i}, \ldots, W_{n}\right]$ are polynomials such that the degree of $f_{i j}$ in $W_{i}$ is $k_{j}$.

Fix $j \in\{1, \ldots, l-1\}$ and $\mathbf{b} \in \mathbb{Z}^{n}$. By Lemma 3.3, we have

$$
\begin{gathered}
\sum_{i \in\left\{i_{1}, \ldots, i_{l}\right\}} f_{i_{l} j}\left(\ell_{i_{l}}, \ldots, \ell_{n}\right) f_{i l}\left(\ell_{i}, \ldots, \ell_{n}\right) M_{i}^{\mathbf{b}} \in U, \\
\sum_{i \in\left\{i_{1}, \ldots, i_{l}\right\}} f_{i_{l} l}\left(\ell_{i_{l}}, \ldots, \ell_{n}\right) f_{i j}\left(\ell_{i}, \ldots, \ell_{n}\right) M_{i}^{\mathbf{b}} \in U .
\end{gathered}
$$

Subtracting yields

$$
\sum_{i \in\left\{i_{1}, \ldots, i_{l}\right\}} g_{i j}\left(\ell_{i}, \ldots, \ell_{n}\right) M_{i}^{\mathbf{b}} \in U
$$


where

$$
\begin{aligned}
& g_{i j}\left(W_{i}, \ldots, W_{n}\right) \\
= & f_{i_{l} l}\left(W_{i_{l}}, \ldots, W_{n}\right) f_{i j}\left(W_{i}, \ldots, W_{n}\right)-f_{i_{l} j}\left(W_{i_{l}}, \ldots, W_{n}\right) f_{i l}\left(W_{i}, \ldots, W_{n}\right) .
\end{aligned}
$$

Note that $g_{i_{l} j}=0$. For every $i \in\left\{i_{1}, \ldots, i_{l-1}\right\}$, we have $i<i_{l}$. Hence, the variable $W_{i}$ does not occur in the polynomials $f_{i_{l} l}\left(W_{i_{l}}, \ldots, W_{n}\right)$ and $f_{i_{l} j}\left(W_{i_{l}}, \ldots, W_{n}\right)$. Since the degree of the polynomials $f_{i j}\left(W_{i}, \ldots, W_{n}\right)$ and $f_{i l}\left(W_{i}, \ldots, W_{n}\right)$ in $W_{i}$ is $k_{j}$ and $k_{l}$, respectively, with $k_{j}>k_{l}$, and the polynomial $f_{i_{l} l}\left(W_{i_{l}}, \ldots, W_{n}\right)$ is nonzero, the degree of the polynomial $g_{i j}\left(W_{i}, \ldots, W_{n}\right)$ in $W_{i}$ is $k_{j}$.

As $j \in\{1, \ldots, l-1\}$ and $\mathbf{b} \in \mathbb{Z}^{n}$ were arbitrary, we have

$$
\sum_{i \in\left\{i_{1}, \ldots, i_{l-1}\right\}} g_{i j}\left(\ell_{i}, \ldots, \ell_{n}\right) M_{i}^{\mathbf{b}} \in U
$$

for every $\mathbf{b} \in \mathbb{Z}^{n}$ and every $j \in\{1, \ldots, l-1\}$. Since the degree of $g_{i j}\left(W_{i}, \ldots, W_{n}\right)$ in $W_{i}$ is $k_{j}, M_{i_{1}}^{\mathbf{b}}, \ldots, M_{i_{l-1}}^{\mathbf{b}}$ satisfy $l-1$ recurrence relations of order $k_{1}>\cdots>k_{l-1}$. We may now use the induction hypothesis and the lemma follows.

The next lemma generalizes the process of solving the system of equations (3.4) in Example 3.1

Lemma 3.5. Let $i \in\{1, \ldots, n\}$ and let $U$ be a subspace of $V$ such that $M_{i}^{b} \in U$ for all but finitely many $\boldsymbol{b} \in \mathbb{Z}^{n}$. If $M_{i}^{\boldsymbol{b}}$ satisfy a recurrence relation, then $M_{i}^{\boldsymbol{b}} \in U$ for all $\boldsymbol{b} \in \mathbb{Z}^{n}$.

Proof. Let

be such that

$$
f\left(W_{i}, \ldots, W_{n}\right)=\sum_{j_{i}, \ldots, j_{n} \geq 0} \lambda_{j_{i}, \ldots, j_{n}} W_{i}^{j_{i}} \ldots W_{n}^{j_{n}}
$$

$$
f\left(\ell_{i}, \ldots, \ell_{n}\right) M_{i}^{\mathbf{b}} \in U
$$

for every $\mathbf{b} \in \mathbb{Z}^{n}$. The set

$$
J=\left\{\left(j_{i}, \ldots, j_{n}\right) \in \mathbb{N}_{0}^{n-i+1} \mid \lambda_{j_{i}, \ldots, j_{n}} \neq 0\right\}
$$

is nonempty and finite. We can write $f$ as

$$
f\left(W_{i}, \ldots, W_{n}\right)=\sum_{\left(j_{i}, \ldots, j_{n}\right) \in J} \lambda_{j_{i}, \ldots, j_{n}} W_{i}^{j_{i}} \ldots W_{n}^{j_{n}} .
$$

Let $\left(\widetilde{j}_{i}, \ldots, \widetilde{j}_{n}\right)$ be a maximal element of $J$ with respect to the lexicographical ordering.

The set $S=\left\{\mathbf{b} \in \mathbb{Z}^{n} \mid M_{i}^{\mathbf{b}} \notin U\right\}$ is finite. Suppose $S$ is nonempty. Define the following strict partial ordering on $S$ :

$$
\mathbf{b} \prec \mathbf{b}^{\prime} \Longleftrightarrow \mathbf{b}_{k}<\mathbf{b}_{k}^{\prime} \text { for the least } k \geq i \text { satisfying } \mathbf{b}_{k} \neq \mathbf{b}_{k}^{\prime} \text {. }
$$

We remark that this ordering is lexicographic in the last $n-i+1$ terms. Let $\widetilde{\mathbf{b}}$ be a maximal element of $S$ with respect to $\prec$.

Set

$$
\mathbf{b}^{\prime}=\widetilde{\mathbf{b}}+\widetilde{j}_{i} \mathbf{e}_{i}+\cdots+\widetilde{j}_{n} \mathbf{e}_{n} .
$$

We claim that there exists a $u \in U$ such that

$$
f\left(\ell_{i}, \ldots, \ell_{n}\right) M_{i}^{\mathbf{b}^{\prime}}=\lambda_{\widetilde{j}_{i}, \ldots, \tilde{j}_{n}} M_{i}^{\widetilde{\mathbf{b}}}+u .
$$


Indeed, by definition of $\ell_{j}$,

$$
\begin{aligned}
f\left(\ell_{i}, \ldots, \ell_{n}\right) M_{i}^{\mathbf{b}^{\prime}} & =\sum_{\left(j_{i}, \ldots, j_{n}\right) \in J} \lambda_{j_{i}, \ldots, j_{n}} \ell_{i}^{j_{i}} \ldots \ell_{n}^{j_{n}} M_{i}^{\mathbf{b}^{\prime}} \\
& =\sum_{\left(j_{i}, \ldots, j_{n}\right) \in J} \lambda_{j_{i}, \ldots, j_{n}} M_{i}^{\mathbf{b}^{\prime}-j_{i} \mathbf{e}_{i}-\cdots-j_{n} \mathbf{e}_{n}} .
\end{aligned}
$$

Take $\left(j_{i}, \ldots, j_{n}\right) \in J$ distinct from $\left(\widetilde{j}_{i}, \ldots, \widetilde{j}_{n}\right)$. Since $\left(\widetilde{j}_{i}, \ldots, \widetilde{j}_{n}\right)$ is a maximal element of $J$ and the lexicographical ordering is linear, we have $j_{k}<\widetilde{j}_{k}$ for the least $k$ satisfying $j_{k} \neq \widetilde{j}_{k}$. Hence

$$
\begin{aligned}
\mathbf{b} & =\mathbf{b}^{\prime}-j_{i} \mathbf{e}_{i}-\cdots-j_{n} \mathbf{e}_{n} \\
& =\widetilde{\mathbf{b}}+\left(\widetilde{j_{i}}-j_{i}\right) \mathbf{e}_{i}+\cdots+\left(\widetilde{j}_{n}-j_{n}\right) \mathbf{e}_{n}
\end{aligned}
$$

satisfies

$$
\widetilde{\mathbf{b}} \prec \mathbf{b}
$$

if $\mathbf{b} \in S$. As $\widetilde{\mathbf{b}}$ is a maximal element of $S$, it follows that $\mathbf{b} \notin S$, i.e., $M_{i}^{\mathbf{b}} \in U$. Thus, all summands of

$$
\sum_{\left(j_{i}, \ldots, j_{n}\right) \in J} \lambda_{j_{i}, \ldots, j_{n}} M_{i}^{\mathbf{b}^{\prime}-j_{i} \mathbf{e}_{i}-\cdots-j_{n} \mathbf{e}_{n}}
$$

except the one corresponding to $\left(\widetilde{j}_{i}, \ldots, \widetilde{j}_{n}\right)$ are elements of $U$. This proves our claim (3.8).

From (3.7) and (3.8) we deduce

$$
\lambda_{\widetilde{j}_{i}, \ldots, \widetilde{j}_{n}} M_{i}^{\widetilde{\mathbf{b}}} \in U
$$

Since $\widetilde{\mathbf{b}} \in S$, we have $M_{i}^{\widetilde{\mathbf{b}}} \notin U$. Thus, $\lambda_{\widetilde{j}_{i}, \ldots, \widetilde{j}_{n}}=0$, which is a contradiction to $\left(\widetilde{j}_{i}, \ldots, \widetilde{j}_{n}\right) \in J$.

Recall that we only need to prove that $U=V$, having in mind that $M_{1}^{\mathbf{b}}, \ldots, M_{n}^{\mathbf{b}}$ satisfy a recurrence relation of order $k$ for each $k \in \mathbb{N}$ and that $M_{i}^{\mathbf{b}} \in U$ holds for all but finitely many elements. This easily follows from repeated use of Lemmas 3.4 and 3.5. Thus, we have proven the following proposition, which concludes this section.

Proposition 3.6. Let $\sigma \in S_{n}$ and $r \in \mathbb{N}_{0}$. If $\mu_{i}^{b^{\prime}} \in F$ are such that $\mu_{i}^{b^{\prime}}=0$ whenever $b^{\prime} \notin \mathscr{B}_{r}$ and

$$
\sum_{i=1}^{n} \sum_{\boldsymbol{c} \in \mathscr{C}_{\sigma, k, i}} \alpha_{\sigma, i}^{c} \mu_{i}^{b-c}=0
$$

for every $k \in \mathbb{N}$ and every $\boldsymbol{b} \in \mathscr{B}_{r+k}$, then $\mu_{i}^{\boldsymbol{b}^{\prime}}=0$ for all $\boldsymbol{b}^{\prime} \in \mathscr{B}_{r}, i=1, \ldots, n$.

\section{MAIN THEOREM}

First, we fix some notation. Throughout this section, we assume that $A$ is a unital algebra containing an element $v$ with the property that

$$
1 \in \bigcap_{k \in \mathbb{N}}[v, A]_{k}
$$

That is to say, for each $k \in \mathbb{N}$ there exists a $z_{k} \in A$ such that

$$
[\underbrace{v, \ldots, v}_{k}, z_{k}]=1
$$


Let us emphasize that this is the only requirement we impose on $A$. Thus, $A$ does not need to have a surjective inner derivation, but only an element $v$ satisfying (4.1) for every $k \in \mathbb{N}$. Observe that under the assumption that $F$ has characteristic 0 it is enough to require only the existence of $z_{1}$, since then $z_{k}=\frac{1}{k !} z_{1}^{k}$ automatically satisfies (4.1) for any $k$.

We will write

$$
A_{v}=\bigcap_{k \in \mathbb{N}}[v, A]_{k}
$$

Thus, our assumption is that $1 \in A_{v}$. Our goal is to prove Theorem 4.7 which states that the image of any nonzero admissible partially commutative polynomial $f$ contains $A_{v}$. If the derivation $x \mapsto[v, x]$ is surjective, then $A_{v}=A$ and so this simply means that $f$ is surjective on $A$.

The proof is by induction on the number of noncommuting variables $X_{1}, \ldots, X_{n}$. We start with the base case $n=1$.

Lemma 4.1. Let $f \in F\left\langle X_{1}\right\rangle \amalg F[U, V]$ be a nonzero admissible partially commutative polynomial. Then $A_{v} \subseteq f(A ; v)$.

Proof. We will only prove the lemma for admissible polynomials of type two; the type one case is similar. Since $n=1, \mathscr{B}_{r}=\{(r)\}$. Thus, $f$ is equal to

$$
f=\lambda X_{1}^{(r), 1}=\lambda\left[U,\left[V, X_{1}\right]_{r}\right]
$$

for some nonzero $\lambda \in F$. Let $a$ be an arbitrary element in $A_{v}$. By the definition of $A_{v}$, there exists an $x \in A$ such that $a=[v, x]_{r+1}$. As the image of $f$ contains

$$
\operatorname{Ev}_{\lambda^{-1} x ; v}(f)=\lambda\left[v,\left[v, \lambda^{-1} x\right]_{r}\right]=a,
$$

$A_{v} \subseteq f(A ; v)$ follows.

To make the induction step, we first record a lemma which will help us reduce the number of noncommuting variables in a suitable way.

Lemma 4.2. Let $f \in F\left\langle X_{1}, \ldots, X_{n}\right\rangle \amalg F[U, V]$ be a polynomial of the form

$$
f=\sum_{\sigma \in S_{n-1}} \sum_{j=1}^{n} \sum_{b \in \mathscr{B}_{r}} \lambda_{\sigma, j}^{b}\left(X_{\sigma(1)} \ldots X_{\sigma(j-1)} X_{n} X_{\sigma(j)} \ldots X_{\sigma(n-1)}\right)^{b},
$$

where $r \in \mathbb{N}_{0}$. Let $k \in\{0,1, \ldots, r\}$ be such that for every $\boldsymbol{b} \in \mathscr{B}_{r}, \boldsymbol{b}_{n}<k$ implies $\lambda_{\sigma, j}^{b}=0$ for every $\sigma \in S_{n-1}$ and every $j \in\{1, \ldots, n\}$. Then the partially commutative polynomial $g \in F\left\langle X_{1}, \ldots, X_{n}\right\rangle \amalg F[U, V]$ defined by

$$
g=\sum_{\sigma \in S_{n-1}} \sum_{j=1}^{n} \sum_{b^{\prime} \in \mathscr{B}_{r}^{\prime}} \lambda_{\sigma, j}^{b^{\prime}}\left(X_{\sigma(1)} \ldots X_{\sigma(j-1)}\right)^{\boldsymbol{b}^{\prime}} U\left(X_{\sigma(j)} \ldots X_{\sigma(n-1)}\right)^{\boldsymbol{b}^{\prime}},
$$

where $\mathscr{B}_{r}^{\prime}=\left\{\boldsymbol{b}^{\prime} \in \mathscr{B}_{r} \mid \boldsymbol{b}_{n}^{\prime}=k\right\}$, satisfies $g(A ; v) \subseteq f(A ; v)$.

Proof. Take $x_{1}, \ldots, x_{n-1} \in A$ and $u \in F[v]$. Let $z_{k} \in A$ be the element satisfying (4.1). We will prove that

$$
\operatorname{Ev}_{x_{1}, \ldots, x_{n-1} ; u}(g)=\operatorname{Ev}_{x_{1}, \ldots, x_{n-1}, z_{k} u}(f),
$$

from which $g(A ; v) \subseteq f(A ; v)$ follows. 
Consider the expression $\operatorname{Ev}_{x_{1}, \ldots, x_{n-1}, z_{k} u ;}\left(\lambda_{\sigma, j}^{\mathbf{b}} X_{n}^{\mathbf{b}}\right)$ with $\mathbf{b} \in \mathscr{B}_{r}, \sigma \in S_{n-1}$, and $j \in\{1, \ldots, n\}$. If $\mathbf{b}_{n}<k$, then $\lambda_{\sigma, j}^{\mathbf{b}}=0$ and so this expression is zero, and if $\mathbf{b}_{n} \geq k$, then it is equal to

$$
\begin{aligned}
\operatorname{Ev}_{x_{1}, \ldots, x_{n-1}, z_{k} u ;}\left(\lambda_{\sigma, j}^{\mathbf{b}} X_{n}^{\mathbf{b}}\right) & =\lambda_{\sigma, j}^{\mathbf{b}} \operatorname{Ev}_{x_{1}, \ldots, x_{n-1}, z_{k} u ;}\left(\left[V, X_{n}\right]_{\mathbf{b}_{n}}\right) \\
& =\lambda_{\sigma, j}^{\mathbf{b}}\left[v, z_{k} u\right]_{\mathbf{b}_{n} .}
\end{aligned}
$$

Since $u$ commutes with $v$, we have

$$
\begin{aligned}
\operatorname{Ev}_{x_{1}, \ldots, x_{n-1}, z_{k} u ;}\left(\lambda_{\sigma, j}^{\mathbf{b}} X_{n}^{\mathbf{b}}\right) & =\lambda_{\sigma, j}^{\mathbf{b}}\left[v, z_{k}\right]_{\mathbf{b}_{n}} u \\
& =\lambda_{\sigma, j}^{\mathbf{b}}[\underbrace{v, \ldots, v}_{\mathbf{b}_{n}-k},\left[v, z_{k}\right]_{k}] u \\
& =\lambda_{\sigma, j}^{\mathbf{b}}[v, 1]_{\mathbf{b}_{n}-k} u,
\end{aligned}
$$

which is zero if $\mathbf{b}_{n}>k$ and equal to $\lambda_{\sigma, j}^{\mathbf{b}} u$ if $\mathbf{b}_{n}=k$. Thus, we have

$$
\operatorname{Ev}_{x_{1}, \ldots, x_{n-1}, z_{k} u ;}\left(\lambda_{\sigma, j}^{\mathbf{b}} X_{n}^{\mathbf{b}}\right)=\left\{\begin{array}{l}
\lambda_{\sigma, j}^{\mathbf{b}} u, \mathbf{b}_{n}=k \\
0, \mathbf{b}_{n} \neq k
\end{array} .\right.
$$

Hence,

$$
\begin{aligned}
& \operatorname{Ev}_{x_{1}, \ldots, x_{n-1}, z_{k} u ;}(f) \\
= & \sum_{\sigma \in S_{n-1}} \sum_{j=1}^{n} \sum_{\mathbf{b} \in \mathscr{B}_{r}} \operatorname{Ev}_{x_{1}, \ldots, x_{n-1}, z_{k} u ;}\left(\left(X_{\sigma(1)} \ldots X_{\sigma(j-1)}\right)^{\mathbf{b}}\right) \operatorname{Ev}_{x_{1}, \ldots, x_{n-1}, z_{k} u ;}\left(\lambda_{\sigma, j}^{\mathbf{b}} X_{n}^{\mathbf{b}}\right) \\
& \cdot \operatorname{Ev}_{x_{1}, \ldots, x_{n-1}, z_{k} u ;}\left(\left(X_{\sigma(j)} \ldots X_{\sigma(n-1)}\right)^{\mathbf{b}}\right) \\
= & \sum_{\sigma \in S_{n-1}} \sum_{j=1}^{n} \sum_{\mathbf{b}^{\prime} \in \mathscr{B}_{r}^{\prime}} \operatorname{Ev}_{x_{1}, \ldots, x_{n-1}, z_{k} u ;}\left(\left(X_{\sigma(1)} \ldots X_{\sigma(j-1)}\right)^{\mathbf{b}^{\prime}}\right) \lambda_{\sigma, j}^{\mathbf{b}^{\prime} u} \\
& \cdot \operatorname{Ev}_{x_{1}, \ldots, x_{n-1}, z_{k} u ;}\left(\left(X_{\sigma(j)} \ldots X_{\sigma(n-1)}\right)^{\mathbf{b}^{\prime}}\right) .
\end{aligned}
$$

Since $u=\operatorname{Ev}_{x_{1}, \ldots, x_{n-1} ; u}(U)$ and the variable $X_{n}$ does not appear in the polynomials $X_{\sigma(1)} \ldots X_{\sigma(j-1)}$ and $X_{\sigma(j)} \ldots X_{\sigma(n-1)}$, we see that $\operatorname{Ev}_{x_{1}, \ldots, x_{n-1}, z_{k} u}(f)$ is equal to

$$
\sum_{\sigma \in S_{n-1}} \sum_{j=1}^{n} \sum_{\mathbf{b}^{\prime} \in \mathscr{B}_{r}^{\prime}} \lambda_{\sigma, j}^{\mathbf{b}^{\prime}} \operatorname{Ev}_{x_{1}, \ldots, x_{n-1} ; u}\left(\left(X_{\sigma(1)} \ldots X_{\sigma(j-1)}\right)^{\mathbf{b}^{\prime}} U\left(X_{\sigma(j)} \ldots X_{\sigma(n-1)}\right)^{\mathbf{b}^{\prime}}\right) \text {, }
$$

which is exactly $\operatorname{Ev}_{x_{1}, \ldots, x_{n-1} ; u}(g)$.

We are now in a position to make the induction step for admissible polynomials of type one.

Lemma 4.3. Suppose $n \geq 2$ is such that $A_{v} \subseteq g(A ; v)$ holds for every nonzero admissible partially commutative polynomial $g \in F\left\langle X_{1}, \ldots, X_{n-1}\right\rangle \amalg F[U, V]$ (of either type one or type two). Then $A_{v} \subseteq f(A ; v)$ for every nonzero admissible partially commutative polynomial $f \in F\left\langle X_{1}, \ldots, X_{n}\right\rangle \amalg F[U, V]$ of type one.

Proof. Suppose the lemma is not true. Then there exists a polynomial $f$ of the form

$$
f=\sum_{\sigma \in S_{n}} \sum_{\mathbf{b} \in \mathscr{B}_{r}} \lambda_{\sigma}^{\mathbf{b}}\left(X_{\sigma(1)} X_{\sigma(2)} \ldots X_{\sigma(n)}\right)^{\mathbf{b}}
$$


where $\lambda_{\sigma}^{\mathbf{b}} \in F$ are not all zero, such that $f(A ; v)$ does not contain $A_{v}$. We rewrite $f$ as

$$
f=\sum_{\sigma \in S_{n-1}} \sum_{j=1}^{n} \sum_{\mathbf{b} \in \mathscr{B}_{r}} \lambda_{\sigma, j}^{\mathbf{b}}\left(X_{\sigma(1)} \ldots X_{\sigma(j-1)} X_{n} X_{\sigma(j)} \ldots X_{\sigma(n-1)}\right)^{\mathbf{b}},
$$

for some $\lambda_{\sigma, j}^{\mathbf{b}} \in F$, not all zero.

The set

$$
K=\left\{k \in \mathbb{N}_{0} \mid \mathbf{b}_{n}=k \text { and } \lambda_{\sigma, j}^{\mathbf{b}} \neq 0 \text { for some } \sigma \in S_{n-1}, \mathbf{b} \in \mathscr{B}_{r}, j=1, \ldots, n\right\}
$$

is nonempty. Let $k$ be the least number in $K$. Then, for every $\mathbf{b} \in \mathscr{B}_{r}, \mathbf{b}_{n}<k$ implies $\lambda_{\sigma, j}^{\mathbf{b}}=0$ for every $\sigma \in S_{n-1}$ and every $j \in\{1, \ldots, n\}$. By Lemma 4.2, $g(A ; v) \subseteq f(A ; v)$ holds for the polynomial $g \in F\left\langle X_{1}, \ldots, X_{n-1}\right\rangle \amalg F[U, V]$ defined by

$$
g=\sum_{\sigma \in S_{n-1}} \sum_{j=1}^{n} \sum_{\mathbf{b}^{\prime} \in \mathscr{B}_{r}^{\prime}} \lambda_{\sigma, j}^{\mathbf{b}^{\prime}}\left(X_{\sigma(1)} \ldots X_{\sigma(j-1)}\right)^{\mathbf{b}^{\prime}} U\left(X_{\sigma(j)} \ldots X_{\sigma(n-1)}\right)^{\mathbf{b}^{\prime}},
$$

where $\mathscr{B}_{r}^{\prime}=\left\{\mathbf{b}^{\prime} \in \mathscr{B}_{r} \mid \mathbf{b}_{n}^{\prime}=k\right\}$. Since the variable $X_{n}$ does not occur in this expression, we can replace $\mathbf{b}^{\prime}$ by the sequence $\mathbf{b}$ consisting of the first $n-1$ terms of $\mathbf{b}^{\prime}$. Such sequences $\mathbf{b}$ are exactly the elements of $\mathscr{B}_{r-k}$. Thus, we have

$$
g=\sum_{\sigma \in S_{n-1}} \sum_{j=1}^{n} \sum_{\mathbf{b} \in \mathscr{B}_{r-k}} \lambda_{\sigma, j}^{\mathbf{b}^{\prime}}\left(X_{\sigma(1)} \ldots X_{\sigma(j-1)}\right)^{\mathbf{b}} U\left(X_{\sigma(j)} \ldots X_{\sigma(n-1)}\right)^{\mathbf{b}},
$$

where $\mathscr{B}_{r-k}$ consists of sequences of length $n-1$ and $\mathbf{b}^{\prime}$ is the sequence of length $n$ obtained by adding $k$ to the end of $\mathbf{b}$.

The polynomial $g$ may not be admissible. By using the homomorphism $\pi_{0}$ (defined in Section 2), we obtain

$$
\pi_{0}(g)=\sum_{\sigma \in S_{n-1}} \sum_{\mathbf{b} \in \mathscr{B}_{r-k}}\left(\sum_{j=1}^{n} \lambda_{\sigma, j}^{\mathbf{b}^{\prime}}\right)\left(X_{\sigma(1)} \ldots X_{\sigma(j-1)} X_{\sigma(j)} \ldots X_{\sigma(n-1)}\right)^{\mathbf{b}} .
$$

As we see, $\pi_{0}(g)$ is an admissible partially commutative polynomial (of type one) in $n-1$ noncommuting variables. Since the image of $\pi_{0}(g)$ is a subset of $f(A ; v)$ (see (2.1) ), it cannot contain $A_{v}$. Hence, by our assumption, $\pi_{0}(g)$ is zero. By Proposition 2.4. $\sum_{j=1}^{n} \lambda_{\sigma, j}^{\mathbf{b}^{\prime}}=0$ for every $\sigma \in S_{n-1}$ and every $\mathbf{b} \in \mathscr{B}_{r-k}$.

Considering $\lambda_{\sigma, n}^{\mathbf{b}^{\prime}}=-\sum_{j=1}^{n-1} \lambda_{\sigma, j}^{\mathbf{b}^{\prime}}$, we can write $g$ as

$$
\begin{array}{r}
g=\sum_{\sigma \in S_{n-1}} \sum_{\mathbf{b} \in \mathscr{B}_{r-k}} \sum_{j=1}^{n-1} \lambda_{\sigma, j}^{\mathbf{b}^{\prime}}\left(\left(X_{\sigma(1)} \ldots X_{\sigma(j-1)}\right)^{\mathbf{b}} U\left(X_{\sigma(j)} \ldots X_{\sigma(n-1)}\right)^{\mathbf{b}}\right. \\
\left.-\left(X_{\sigma(1)} \ldots X_{\sigma(j-1)} X_{\sigma(j)} \ldots X_{\sigma(n-1)}\right)^{\mathbf{b}} U\right)
\end{array}
$$

which further equals

$$
g=\sum_{\sigma \in S_{n-1}} \sum_{\mathbf{b} \in \mathscr{B}_{r-k}} \sum_{j=1}^{n-1} \lambda_{\sigma, j}^{\mathbf{b}^{\prime}}\left(X_{\sigma(1)} \ldots X_{\sigma(j-1)}\right)^{\mathbf{b}}\left[U,\left(X_{\sigma(j)} \ldots X_{\sigma(n-1)}\right)^{\mathbf{b}}\right] .
$$


Making use of the well-known formula $[X, Y Z]=[X, Y] Z+Y[X, Z]$, we see that

$$
\begin{aligned}
& {\left[U,\left(X_{\sigma(j)} \ldots X_{\sigma(n-1)}\right)^{\mathbf{b}}\right] } \\
= & \sum_{i=j}^{n-1}\left(X_{\sigma(j)} \ldots X_{\sigma(i-1)}\right)^{\mathbf{b}}\left[U, X_{\sigma(i)}^{\mathbf{b}}\right]\left(X_{\sigma(i+1)} \ldots X_{\sigma(n-1)}\right)^{\mathbf{b}} .
\end{aligned}
$$

Since

$$
\left[U, X_{\sigma(i)}^{\mathbf{b}}\right]=X_{\sigma(i)}^{\mathbf{b}, \sigma(i)}
$$

we have

$$
\left[U,\left(X_{\sigma(j)} \ldots X_{\sigma(n-1)}\right)^{\mathbf{b}}\right]=\sum_{i=j}^{n-1}\left(X_{\sigma(j)} \ldots X_{\sigma(n-1)}\right)^{\mathbf{b}, \sigma(i)}
$$

Thus,

$$
\begin{aligned}
g & =\sum_{\sigma \in S_{n-1}} \sum_{\mathbf{b} \in \mathscr{B}_{r-k}} \sum_{j=1}^{n-1} \lambda_{\sigma, j}^{\mathbf{b}^{\prime}}\left(X_{\sigma(1)} \ldots X_{\sigma(j-1)}\right)^{\mathbf{b}} \sum_{i=j}^{n-1}\left(X_{\sigma(j)} \ldots X_{\sigma(n-1)}\right)^{\mathbf{b}, \sigma(i)} \\
& =\sum_{\sigma \in S_{n-1}} \sum_{\mathbf{b} \in \mathscr{B}_{r-k}} \sum_{j=1}^{n-1} \sum_{i=j}^{n-1} \lambda_{\sigma, j}^{\mathbf{b}^{\prime}}\left(X_{\sigma(1)} \ldots X_{\sigma(n-1)}\right)^{\mathbf{b}, \sigma(i)} .
\end{aligned}
$$

By changing the order of summation, we obtain

$$
g=\sum_{\sigma \in S_{n-1}} \sum_{\mathbf{b} \in \mathscr{B}_{r-k}} \sum_{i=1}^{n-1}\left(\sum_{j=1}^{i} \lambda_{\sigma, j}^{\mathbf{b}^{\prime}}\right)\left(X_{\sigma(1)} \ldots X_{\sigma(n-1)}\right)^{\mathbf{b}, \sigma(i)} .
$$

As we can see, $g$ is an admissible partially commutative polynomial (of type two) in $n-1$ noncommuting variables. Since the image of $g$ is a subset of $f(A ; v)$, it cannot contain $A_{v}$. Hence, by our assumption, $g$ is zero. By Proposition 2.4, we have $\sum_{j=1}^{i} \lambda_{\sigma, j}^{\mathbf{b}^{\prime}}=0$ for every $\sigma \in S_{n-1}$, every $\mathbf{b} \in \mathscr{B}_{r-k}$, and every $i=1, \ldots, n$ (the $i=n$ case follows from the first part of the proof). This obviously implies that $\lambda_{\sigma, j}^{\mathbf{b}^{\prime}}=0$ for every $\sigma \in S_{n-1}$, every $\mathbf{b}^{\prime} \in \mathscr{B}_{r}^{\prime}$, and every $j=1, \ldots, n$. However, this is in contradiction with $k \in K$.

It remains to consider admissible polynomials of type two. The main idea of our approach is to use Lemma 4.3 by applying the homomorphism $\pi_{k}$. Since the image of $\pi_{k}$ of an admissible polynomial is not necessarily admissible, our goal is to write $\pi_{k}\left(\left(X_{\sigma(1)} \ldots X_{\sigma(n)}\right)^{\mathbf{b}, \sigma(i)}\right)$ as a linear combination of the polynomials $\left(X_{\sigma(1)} \ldots X_{\sigma(n)}\right)^{\mathbf{b}+\mathbf{c}} V^{t}$ for some sequences $\mathbf{c}$ and nonnegative integers $t$. The following lemmas are devoted to this purpose. We remark that our calculations are based on

$$
V X_{i}^{\mathbf{b}}=\left[V, X_{i}^{\mathbf{b}}\right]+X_{i}^{\mathbf{b}} V=X_{i}^{\mathbf{b}+\mathbf{e}_{i}}+X_{i}^{\mathbf{b}} V .
$$

Lemma 4.4. For any $k \in \mathbb{N}$, we have

$$
\pi_{k}\left(X_{i}^{\boldsymbol{b}, i}\right)=\sum_{s=1}^{k}\left(\begin{array}{l}
k \\
s
\end{array}\right) X_{i}^{\boldsymbol{b}+s \boldsymbol{e}_{i}} V^{k-s} .
$$


Proof. We proceed by induction on $k$. Since $\pi_{1}\left(X_{i}^{\mathbf{b}, i}\right)=X_{i}^{\mathbf{b}+\mathbf{e}_{i}}$, the lemma is obviously true for $k=1$, so assume that $k>1$ and that the lemma is true for $k-1$. We have

$$
\pi_{k}\left(X_{i}^{\mathbf{b}, i}\right)=\left[V^{k}, X_{i}^{\mathbf{b}}\right]=V\left[V^{k-1}, X_{i}^{\mathbf{b}}\right]+\left[V, X_{i}^{\mathbf{b}}\right] V^{k-1} .
$$

As $\left[V^{k-1}, X_{i}^{\mathbf{b}}\right]=\pi_{k-1}\left(X_{i}^{\mathbf{b}, i}\right)$, it follows from the induction hypothesis that

$$
\pi_{k}\left(X_{i}^{\mathbf{b}, i}\right)=V \sum_{s=1}^{k-1}\left(\begin{array}{c}
k-1 \\
s
\end{array}\right) X_{i}^{\mathbf{b}+s \mathbf{e}_{i}} V^{k-1-s}+X_{i}^{\mathbf{b}+\mathbf{e}_{i}} V^{k-1} .
$$

Using (4.2), it follows that

$$
\begin{aligned}
\pi_{k}\left(X_{i}^{\mathbf{b}, i}\right)= & \sum_{s=1}^{k-1}\left(\begin{array}{c}
k-1 \\
s
\end{array}\right) X_{i}^{\mathbf{b}+(s+1) \mathbf{e}_{i}} V^{k-1-s}+\sum_{s=1}^{k-1}\left(\begin{array}{c}
k-1 \\
s
\end{array}\right) X_{i}^{\mathbf{b}+s \mathbf{e}_{i}} V^{k-s} \\
& +X_{i}^{\mathbf{b}+\mathbf{e}_{i}} V^{k-1} .
\end{aligned}
$$

By changing the index of summation in the first sum and splitting the second sum, we obtain

$$
\begin{aligned}
\pi_{k}\left(X_{i}^{\mathbf{b}, i}\right)= & \sum_{s=2}^{k}\left(\begin{array}{c}
k-1 \\
s-1
\end{array}\right) X_{i}^{\mathbf{b}+s \mathbf{e}_{i}} V^{k-s}+\sum_{s=2}^{k-1}\left(\begin{array}{c}
k-1 \\
s
\end{array}\right) X_{i}^{\mathbf{b}+s \mathbf{e}_{i}} V^{k-s} \\
& +k X_{i}^{\mathbf{b}+\mathbf{e}_{i}} V^{k-1}
\end{aligned}
$$

and hence

$$
\begin{aligned}
\pi_{k}\left(X_{i}^{\mathbf{b}, i}\right)= & X_{i}^{\mathbf{b}+k \mathbf{e}_{i}}+\sum_{s=2}^{k-1}\left(\left(\begin{array}{c}
k-1 \\
s-1
\end{array}\right)+\left(\begin{array}{c}
k-1 \\
s
\end{array}\right)\right) X_{i}^{\mathbf{b}+s \mathbf{e}_{i}} V^{k-s} \\
& +k X_{i}^{\mathbf{b}+\mathbf{e}_{i}} V^{k-1}
\end{aligned}
$$

Using

$$
\left(\begin{array}{c}
k-1 \\
s-1
\end{array}\right)+\left(\begin{array}{c}
k-1 \\
s
\end{array}\right)=\left(\begin{array}{l}
k \\
s
\end{array}\right)
$$

we obtain the conclusion of the lemma.

We introduce the set

$$
\mathscr{D}_{t, i}=\left\{\mathbf{d}=\left(\mathbf{d}_{1}, \ldots, \mathbf{d}_{n}\right) \in \mathbb{N}_{0}^{n} \mid \mathbf{d}_{1}=\ldots=\mathbf{d}_{i-1}=0, \sum_{j=1}^{n} \mathbf{d}_{j}=t\right\} .
$$

Note that $\mathscr{D}_{t, i}$ is in bijective correspondence with $\sqcup_{s=0}^{t} \mathscr{D}_{t-s, i+1}$, the disjoint union of the sets $\mathscr{D}_{t-s, i+1}$, via $\mathbf{d}=s \mathbf{e}_{i}+\mathbf{d}^{\prime}$. We will use this in the proof of the following lemma.

Lemma 4.5. For any $k \in \mathbb{N}$ and $s \in\{1, \ldots, k\}$, we have

$$
V^{k-s}\left(X_{i+1} \ldots X_{n}\right)^{\boldsymbol{b}}=\sum_{t=s}^{k}\left(\begin{array}{c}
k-s \\
t-s
\end{array}\right) \sum_{\boldsymbol{d} \in \mathscr{D}_{t-s, i+1}}\left(\begin{array}{c}
t-s \\
\boldsymbol{d}_{i+1}, \ldots, \boldsymbol{d}_{n}
\end{array}\right)\left(X_{i+1} \ldots X_{n}\right)^{\boldsymbol{b}+\boldsymbol{d}} V^{k-t} .
$$


Proof. We proceed by induction on $n-i$. For $n-i=1$, we see by repeated use of (4.2) that

$$
V^{k-s} X_{n}^{\mathbf{b}}=\sum_{t=0}^{k-s}\left(\begin{array}{c}
k-s \\
t
\end{array}\right) X_{n}^{b+t \mathbf{e}_{n}} V^{k-s-t} .
$$

By changing the index of summation and considering $\mathscr{D}_{t-s, n}=\left\{(t-s) \mathbf{e}_{n}\right\}$, we see that this is further equal to

$$
V^{k-s} X_{n}^{\mathbf{b}}=\sum_{t=s}^{k}\left(\begin{array}{c}
k-s \\
t-s
\end{array}\right) \sum_{\mathbf{d} \in \mathscr{D}_{t-s, n}}\left(\begin{array}{c}
t-s \\
\mathbf{d}_{n}
\end{array}\right) X_{n}^{\mathbf{b}+\mathbf{d}} V^{k-t},
$$

which proves the base case.

Now let $n-i>1$ and assume that the lemma is true for all numbers smaller than $n-i$. Use (4.3) and the induction hypothesis to obtain

$$
\begin{aligned}
& V^{k-s}\left(X_{i+1} \ldots X_{n}\right)^{\mathbf{b}} \\
= & \sum_{s^{\prime}=0}^{k-s}\left(\begin{array}{c}
k-s \\
s^{\prime}
\end{array}\right) X_{i+1}^{\mathbf{b}+s^{\prime} \mathbf{e}_{i+1}} V^{k-s-s^{\prime}}\left(X_{i+2} \ldots X_{n}\right)^{\mathbf{b}} \\
= & \sum_{s^{\prime}=0}^{k-s}\left(\begin{array}{c}
k-s \\
s^{\prime}
\end{array}\right) X_{i+1}^{\mathbf{b}+s^{\prime} \mathbf{e}_{i+1}} \\
& \cdot \sum_{t=s+s^{\prime}}^{k}\left(\begin{array}{c}
k-s-s^{\prime} \\
t-s-s^{\prime}
\end{array}\right) \sum_{\mathbf{d} \in \mathscr{D}_{t-s-s^{\prime}, i+2}}\left(\begin{array}{c}
t-s-s^{\prime} \\
\mathbf{d}_{i+2}, \ldots, \mathbf{d}_{n}
\end{array}\right)\left(X_{i+2} \ldots X_{n}\right)^{\mathbf{b}+\mathbf{d}} V^{k-t} .
\end{aligned}
$$

Since

$$
\left(\begin{array}{c}
k-s \\
s^{\prime}
\end{array}\right)\left(\begin{array}{c}
k-s-s^{\prime} \\
t-s-s^{\prime}
\end{array}\right)\left(\begin{array}{c}
t-s-s^{\prime} \\
\mathbf{d}_{i+2}, \ldots, \mathbf{d}_{n}
\end{array}\right)=\left(\begin{array}{c}
k-s \\
t-s
\end{array}\right)\left(\begin{array}{c}
t-s \\
s^{\prime}, \mathbf{d}_{i+2}, \ldots, \mathbf{d}_{n}
\end{array}\right)
$$

we have

$$
\begin{aligned}
& V^{k-s}\left(X_{i+1} \ldots X_{n}\right)^{\mathbf{b}} \\
= & \sum_{s^{\prime}=0}^{k-s} \sum_{t=s+s^{\prime}}^{k}\left(\begin{array}{c}
k-s \\
t-s
\end{array}\right) \sum_{\mathbf{d} \in \mathscr{D}_{t-s-s^{\prime}, i+2}}\left(\begin{array}{c}
t-s \\
s^{\prime}, \mathbf{d}_{i+2}, \ldots, \mathbf{d}_{n}
\end{array}\right)\left(X_{i+1} \ldots X_{n}\right)^{\mathbf{b}+s^{\prime} \mathbf{e}_{i+1}+\mathbf{d}} V^{k-t} .
\end{aligned}
$$

Change the order of summation and use the aforementioned bijective correspondence between $\mathscr{D}_{t-s, i+1}$ and $\sqcup_{s^{\prime}=0}^{t-s} \mathscr{D}_{t-s-s^{\prime}, i+2}$ to obtain

$$
\begin{aligned}
& V^{k-s}\left(X_{i+1} \ldots X_{n}\right)^{\mathbf{b}} \\
= & \sum_{t=s}^{k}\left(\begin{array}{c}
k-s \\
t-s
\end{array}\right) \sum_{s^{\prime}=0}^{t-s} \sum_{\mathbf{d} \in \mathscr{D}_{t-s-s^{\prime}, i+2}}\left(\begin{array}{c}
t-s \\
s^{\prime}, \mathbf{d}_{i+2}, \ldots, \mathbf{d}_{n}
\end{array}\right)\left(X_{i+1} \ldots X_{n}\right)^{\mathbf{b}+s^{\prime} \mathbf{e}_{i+1}+\mathbf{d}} V^{k-t} \\
= & \sum_{t=s}^{k}\left(\begin{array}{c}
k-s \\
t-s
\end{array}\right) \sum_{\mathbf{d} \in \mathscr{D}_{t-s, i+1}}\left(\begin{array}{c}
t-s \\
\mathbf{d}_{i+1}, \mathbf{d}_{i+2}, \ldots, \mathbf{d}_{n}
\end{array}\right)\left(X_{i+1} \ldots X_{n}\right)^{\mathbf{b}+\mathbf{d}} V^{k-t},
\end{aligned}
$$

which concludes the induction step. 
Recall from Section 3 that

$$
\mathscr{C}_{\sigma, t, i}=\left\{\mathbf{c}=\left(\mathbf{c}_{1}, \ldots, \mathbf{c}_{n}\right) \in \mathbb{N}_{0}^{n} \mid \mathbf{c}_{\sigma(1)}=\ldots=\mathbf{c}_{\sigma(i-1)}=0, \mathbf{c}_{\sigma(i)} \geq 1, \sum_{j=1}^{n} \mathbf{c}_{j}=t\right\} .
$$

The set $\mathscr{C}_{\text {id }, t, i}$ is in bijective correspondence with $\sqcup_{s=1}^{t} \mathscr{D}_{t-s, i+1}$ via $\mathbf{c}=s \mathbf{e}_{i}+\mathbf{d}$. Recall also that

$$
\alpha_{\sigma, i}^{\mathbf{c}}=\left(\begin{array}{c}
\sum_{j=1}^{n} \mathbf{c}_{j} \\
\mathbf{c}_{\sigma(i)}, \ldots, \mathbf{c}_{\sigma(n)}
\end{array}\right)
$$

and set

$$
h_{\sigma, i, t}^{\mathbf{b}}=\sum_{\mathbf{c} \in \mathscr{C}_{\sigma, t, i}} \alpha_{\sigma, i}^{\mathbf{c}}\left(X_{\sigma(1)} \ldots X_{\sigma(n)}\right)^{\mathbf{b}+\mathbf{c}}
$$

Lemma 4.6. For any $r \in \mathbb{N}_{0}, \sigma \in S_{n}, \boldsymbol{b} \in \mathscr{B}_{r}, i \in\{1, \ldots, n\}$, and $k \in \mathbb{N}$, we have

$$
\pi_{k}\left(\left(X_{\sigma(1)} \ldots X_{\sigma(n)}\right)^{\boldsymbol{b}, \sigma(i)}\right)=\sum_{t=1}^{k}\left(\begin{array}{l}
k \\
t
\end{array}\right) h_{\sigma, i, t}^{\boldsymbol{b}} V^{k-t} .
$$

Proof. We will prove the lemma only for the case where $\sigma=$ id (if $\sigma \neq$ id, just permute the indices in the formulas that follow). First, we write

$$
\pi_{k}\left(\left(X_{1} \ldots X_{n}\right)^{\mathbf{b}, i}\right)=\left(X_{1} \ldots X_{i-1}\right)^{\mathbf{b}} \pi_{k}\left(X_{i}^{\mathbf{b}, i}\right)\left(X_{i+1} \ldots X_{n}\right)^{\mathbf{b}}
$$

and apply Lemma 4.4 to obtain

$$
\pi_{k}\left(\left(X_{1} \ldots X_{n}\right)^{\mathbf{b}, i}\right)=\left(X_{1} \ldots X_{i-1}\right)^{\mathbf{b}} \sum_{s=1}^{k}\left(\begin{array}{c}
k \\
s
\end{array}\right) X_{i}^{\mathbf{b}+s \mathbf{e}_{i}} V^{k-s}\left(X_{i+1} \ldots X_{n}\right)^{\mathbf{b}} .
$$

By Lemma 4.5,

$$
\begin{aligned}
\pi_{k}\left(\left(X_{1} \ldots X_{n}\right)^{\mathbf{b}, i}\right)= & \left(X_{1} \ldots X_{i-1}\right)^{\mathbf{b}} \sum_{s=1}^{k}\left(\begin{array}{l}
k \\
s
\end{array}\right) X_{i}^{\mathbf{b}+s \mathbf{e}_{i}} \\
& \cdot \sum_{t=s}^{k}\left(\begin{array}{c}
k-s \\
t-s
\end{array}\right) \sum_{\mathbf{d} \in \mathscr{D}_{t-s, i+1}}\left(\begin{array}{c}
t-s \\
\mathbf{d}_{i+1}, \ldots, \mathbf{d}_{n}
\end{array}\right)\left(X_{i+1} \ldots X_{n}\right)^{\mathbf{b}+\mathbf{d}} V^{k-t} .
\end{aligned}
$$

Applying (4.4), we have

$$
\begin{aligned}
& \pi_{k}\left(\left(X_{1} \ldots X_{n}\right)^{\mathbf{b}, i}\right) \\
= & \sum_{s=1}^{k} \sum_{t=s}^{k}\left(\begin{array}{l}
k \\
t
\end{array}\right) \sum_{\mathbf{d} \in \mathscr{D}_{t-s, i+1}}\left(\begin{array}{c}
t \\
s, \mathbf{d}_{i+1}, \ldots, \mathbf{d}_{n}
\end{array}\right)\left(X_{1} \ldots X_{n}\right)^{\mathbf{b}+s \mathbf{e}_{i}+\mathbf{d}} V^{k-t} .
\end{aligned}
$$


Change the order of summation and use the aforementioned bijective correspondence between $\mathscr{C}_{\mathrm{id}, t, i}$ and $\sqcup_{s=1}^{t} \mathscr{D}_{t-s, i+1}$ to obtain

$$
\begin{aligned}
& \pi_{k}\left(\left(X_{1} \ldots X_{n}\right)^{\mathbf{b}, i}\right) \\
= & \sum_{t=1}^{k}\left(\begin{array}{l}
k \\
t
\end{array}\right) \sum_{s=1}^{t} \sum_{\mathbf{d} \in \mathscr{D}_{t-s, i+1}}\left(\begin{array}{c}
t \\
s, \mathbf{d}_{i+1}, \ldots, \mathbf{d}_{n}
\end{array}\right)\left(X_{1} \ldots X_{n}\right)^{\mathbf{b}+s \mathbf{e}_{i}+\mathbf{d}} V^{k-t} \\
= & \sum_{t=1}^{k}\left(\begin{array}{l}
k \\
t
\end{array}\right) \sum_{\mathbf{c} \in \mathscr{C}_{\text {id }, t, i}}\left(\begin{array}{c}
t \\
\mathbf{c}_{i}, \mathbf{c}_{i+1}, \ldots, \mathbf{c}_{n}
\end{array}\right)\left(X_{1} \ldots X_{n}\right)^{\mathbf{b}+\mathbf{c}} V^{k-t}
\end{aligned}
$$

which concludes this proof.

Theorem 4.7. Let $A$ be a unital algebra over a field $F$. Suppose $A$ contains an element $v$ such that $1 \in A_{v}=\bigcap_{k \in \mathbb{N}}[v, A]_{k}$. Then $A_{v} \subseteq f(A ; v)$ for every nonzero admissible partially commutative polynomial $f$.

Proof. We proceed by induction on $n$, i.e., the number of noncommuting variables $X_{1}, \ldots, X_{n}$ involved in $f$. The case where $n=1$ was considered in Lemma 4.1 .

Let $n>1$. Assume the theorem is true for all nonzero admissible partially commutative polynomials in $n-1$ noncommuting variables. In light of Lemma 4.3, it suffices to prove that the theorem is true for every nonzero admissible partially commutative polynomial of type two in $n$ noncommuting variables, i.e., a polynomial of the form

$$
f=\sum_{\sigma \in S_{n}} \sum_{\mathbf{b} \in \mathscr{B}_{r}} \sum_{i=1}^{n} \lambda_{\sigma, i}^{\mathbf{b}}\left(X_{\sigma(1)} \ldots X_{\sigma(n)}\right)^{\mathbf{b}, \sigma(i)}
$$

for some $\lambda_{\sigma, i}^{\mathbf{b}} \in F$, not all zero, and some $r \in \mathbb{N}_{0}$.

Suppose that the image of $f$ does not contain $A_{v}$. We claim that

$$
\sum_{\sigma \in S_{n}} \sum_{\mathbf{b} \in \mathscr{B}_{r}} \sum_{i=1}^{n} \lambda_{\sigma, i}^{\mathbf{b}} h_{\sigma, i, k}^{\mathbf{b}}=0
$$

where $h_{\sigma, i, k}^{\mathbf{b}}$ are as in the preceding lemma, holds for every $k \in \mathbb{N}$. Let us prove this by induction on $k$. Since the image of $f$ does not contain $A_{v}$, the same applies to the image of $\pi_{1}(f)$. We have

$$
\pi_{1}(f)=\sum_{\sigma \in S_{n}} \sum_{\mathbf{b} \in \mathscr{B}_{r}} \sum_{i=1}^{n} \lambda_{\sigma, i}^{\mathbf{b}} \pi_{1}\left(\left(X_{\sigma(1)} \ldots X_{\sigma(n)}\right)^{\mathbf{b}, \sigma(i)}\right),
$$

which is, by Lemma 4.6, equal to

$$
\pi_{1}(f)=\sum_{\sigma \in S_{n}} \sum_{\mathbf{b} \in \mathscr{B}_{r}} \sum_{i=1}^{n} \lambda_{\sigma, i}^{\mathbf{b}} h_{\sigma, i, 1}^{\mathbf{b}} .
$$

Since $h_{\sigma, i, 1}^{\mathbf{b}}=\left(X_{\sigma(1)} \ldots X_{\sigma(n)}\right)^{\mathbf{b}+\mathbf{c}}$ and, for $\mathbf{b} \in \mathscr{B}_{r}$ and $\mathbf{c} \in \mathscr{C}_{\sigma, 1, i}=\left\{\mathbf{e}_{\sigma(i)}\right\}$, the sequence $\mathbf{b}+\mathbf{c}$ is an element of $\mathscr{B}_{r+1}, \pi_{1}(f)$ is an admissible partially commutative polynomial of type one in $n$ noncommuting variables. Since the image of $\pi_{1}(f)$ does not contain $A_{v}, \pi_{1}(f)=0$ by Lemma 4.3. This proves (4.5) for $k=1$.

Now, let $k>1$ and assume that (4.5) holds for all positive integers smaller than $k$. Since the image of $f$ does not contain $A_{v}$, the same applies to the image of $\pi_{k}(f)$. 
We have

$$
\pi_{k}(f)=\sum_{\sigma \in S_{n}} \sum_{\mathbf{b} \in \mathscr{B}_{r}} \sum_{i=1}^{n} \lambda_{\sigma, i}^{\mathbf{b}} \pi_{k}\left(\left(X_{\sigma(1)} \ldots X_{\sigma(n)}\right)^{\mathbf{b}, \sigma(i)}\right),
$$

which is, by Lemma 4.6, equal to

$$
\begin{aligned}
\pi_{k}(f) & =\sum_{\sigma \in S_{n}} \sum_{\mathbf{b} \in \mathscr{B}_{r}} \sum_{i=1}^{n} \lambda_{\sigma, i}^{\mathbf{b}} \sum_{t=1}^{k}\left(\begin{array}{l}
k \\
t
\end{array}\right) h_{\sigma, i, t}^{\mathbf{b}} V^{k-t} \\
& =\sum_{t=1}^{k}\left(\begin{array}{l}
k \\
t
\end{array}\right)\left(\sum_{\sigma \in S_{n}} \sum_{\mathbf{b} \in \mathscr{B}_{r}} \sum_{i=1}^{n} \lambda_{\sigma, i}^{\mathbf{b}} h_{\sigma, i, t}^{\mathbf{b}}\right) V^{k-t} .
\end{aligned}
$$

The expression in parenthesis is equal to the left-hand side of (4.5). By the induction hypothesis, we have

$$
\begin{aligned}
\pi_{k}(f) & =\left(\begin{array}{l}
k \\
k
\end{array}\right)\left(\sum_{\sigma \in S_{n}} \sum_{\mathbf{b} \in \mathscr{B}_{r}} \sum_{i=1}^{n} \lambda_{\sigma, i}^{\mathbf{b}} h_{\sigma, i, k}^{\mathbf{b}}\right) V^{k-k} \\
& =\sum_{\sigma \in S_{n}} \sum_{\mathbf{b} \in \mathscr{B}_{r}} \sum_{i=1}^{n} \lambda_{\sigma, i}^{\mathbf{b}} h_{\sigma, i, k}^{\mathbf{b}} .
\end{aligned}
$$

Since $h_{\sigma, i, k}^{\mathbf{b}}$ is a linear combination of the polynomials $\left(X_{\sigma(1)} \ldots X_{\sigma(n)}\right){ }^{\mathbf{b}+\mathbf{c}}$ and, for $\mathbf{b} \in \mathscr{B}_{r}$ and $\mathbf{c} \in \mathscr{C}_{\sigma, k, i}$, the sequence $\mathbf{b}+\mathbf{c}$ is an element of $\mathscr{B}_{r+k}, \pi_{k}(f)$ is an admissible partially commutative polynomial of type one in $n$ noncommuting variables. Since the image of $\pi_{k}(f)$ does not contain $A_{v}, \pi_{k}(f)=0$ by Lemma 4.3. This concludes our induction step. Thus, we have proven (4.5) for every $k \in \mathbb{N}$.

Using the definition of $h_{\sigma, i, k}^{\mathbf{b}}$ in (4.5) we obtain

$$
\sum_{\sigma \in S_{n}} \sum_{\mathbf{b} \in \mathscr{B}_{r}} \sum_{i=1}^{n} \sum_{\mathbf{c} \in \mathscr{C}_{\sigma, k, i}} \lambda_{\sigma, i}^{\mathbf{b}} \alpha_{\sigma, i}^{\mathbf{c}}\left(X_{\sigma(1)} \ldots X_{\sigma(n)}\right)^{\mathbf{b}+\mathbf{c}}=0
$$

that is,

$$
\sum_{\sigma \in S_{n}} \sum_{i=1}^{n} \sum_{\mathbf{c} \in \mathscr{C}_{\sigma, k, i}} \alpha_{\sigma, i}^{\mathbf{c}} \sum_{\mathbf{b} \in \mathscr{B}_{r}} \lambda_{\sigma, i}^{\mathbf{b}}\left(X_{\sigma(1)} \ldots X_{\sigma(n)}\right)^{\mathbf{b}+\mathbf{c}}=0 .
$$

By changing the index of summation to $\mathbf{b}^{\prime}=\mathbf{b}+\mathbf{c}$, we have

$$
\sum_{\mathbf{b} \in \mathscr{B}_{r}} \lambda_{\sigma, i}^{\mathbf{b}}\left(X_{\sigma(1)} \ldots X_{\sigma(n)}\right)^{\mathbf{b}+\mathbf{c}}=\sum_{\mathbf{b}^{\prime} \in \mathscr{B}_{r}+\mathbf{c}} \lambda_{\sigma, i}^{\mathbf{b}^{\prime}-\mathbf{c}}\left(X_{\sigma(1)} \ldots X_{\sigma(n)}\right)^{\mathbf{b}^{\prime}} .
$$

Since $\mathscr{B}_{r}+\mathbf{c}$ is a subset of $\mathscr{B}_{r+k}$, we further have

$$
\sum_{\mathbf{b} \in \mathscr{B}_{r}} \lambda_{\sigma, i}^{\mathbf{b}}\left(X_{\sigma(1)} \ldots X_{\sigma(n)}\right)^{\mathbf{b}+\mathbf{c}}=\sum_{\mathbf{b}^{\prime} \in \mathscr{B}_{r+k}} \mu_{\sigma, i}^{\mathbf{b}^{\prime}-\mathbf{c}}\left(X_{\sigma(1)} \ldots X_{\sigma(n)}\right)^{\mathbf{b}^{\prime}},
$$

where

$$
\mu_{\sigma, i}^{\mathbf{b}}=\left\{\begin{array}{l}
\lambda_{\sigma, i}^{\mathbf{b}}, \mathbf{b} \in \mathscr{B}_{r} \\
0, \mathbf{b} \notin \mathscr{B}_{r}
\end{array} .\right.
$$


Using (4.7) in (4.6) we get

$$
\sum_{\sigma \in S_{n}} \sum_{i=1}^{n} \sum_{\mathbf{c} \in \mathscr{C}_{\sigma, k, i}} \alpha_{\sigma, i}^{\mathbf{c}} \sum_{\mathbf{b}^{\prime} \in \mathscr{B}_{r+k}} \mu_{\sigma, i}^{\mathbf{b}^{\prime}-\mathbf{c}}\left(X_{\sigma(1)} \ldots X_{\sigma(n)}\right)^{\mathbf{b}^{\prime}}=0
$$

or, equivalently,

$$
\sum_{\sigma \in S_{n}} \sum_{\mathbf{b}^{\prime} \in \mathscr{B}_{r+k}}\left(\sum_{i=1}^{n} \sum_{\mathbf{c} \in \mathscr{C}_{\sigma, k, i}} \alpha_{\sigma, i}^{\mathbf{c}} \mu_{\sigma, i}^{\mathbf{b}^{\prime}-\mathbf{c}}\right)\left(X_{\sigma(1)} \ldots X_{\sigma(n)}\right)^{\mathbf{b}^{\prime}}=0 .
$$

By Proposition 2.4.

$$
\sum_{i=1}^{n} \sum_{\mathbf{c} \in \mathscr{C}_{\sigma, k, i}} \alpha_{\sigma, i}^{\mathbf{c}} \mu_{\sigma, i}^{\mathbf{b}^{\prime}-\mathbf{c}}=0
$$

for every $\sigma \in S_{n}$, every $k \in \mathbb{N}$, and every $\mathbf{b}^{\prime} \in \mathscr{B}_{r+k}$. Now, Proposition 3.6 tells us that each $\mu_{\sigma, i}^{\mathbf{b}^{\prime}-\mathbf{c}}$ is zero, and consequently, each $\lambda_{\sigma, i}^{\mathbf{b}}$ is zero. We have thus arrived at a contradiction to the assumption that $f \neq 0$.

Finally, we point out that since multilinear noncommutative polynomials are special examples of admissible partially commutative polynomials (see Remark 2.2), Theorem 1.2 follows immediately from Theorem 4.7 .

\section{ACKNOWLEDGMENT}

The author would like to thank his supervisor Matej Brešar for his constant guidance, support, and tireless efforts in improving this paper.

\section{REFERENCES}

[1] K. I. Beidar, W. S. Martindale 3rd, A. V. Mikhalev, Rings with generalized identities, Marcel Dekker, Inc., 1996.

[2] M. Brešar, Commutators and images of noncommutative polynomials, Adv. Math. 374 (2020), 107346.

[3] P. M. Cohn, The range of derivations on a skew field and the equation $a x-x b=c$, J. Indian Math. Soc. 37 (1973), 61-69.

[4] A. Kanel-Belov, No associative PI-algebra coincides with its commutant, Siberian Math. J. 44 (2003), 969-980.

[5] A. Kanel-Belov, S. Malev, L. Rowen, The images of non-commutative polynomials evaluated on $2 \times 2$ matrices, Proc. Amer. Math. Soc. 140 (2012), 465-478.

[6] L. Rowen, R. Yavich, S. Malev, A. Kanel-Belov, Evaluations of noncommutative polynomials on algebras: methods and problems, and the L'vov-Kaplansky conjecture, SIGMA 16 (2020), 071.

Department of Mathematics, Faculty of Mathematics and Physics, University of Luubluana, Slovenia

Email address: daniel.vitas@student.fmf.uni-lj.si 